\title{
Clinical effects of low-molecular-weight heparin combined with ulinastatin in children with acute pancreatitis
}

\author{
Ai-Hua $\operatorname{Han}^{1 *}$, Guo-Qing $\mathrm{Yu}^{1}$ and Hua-Zhen $\mathrm{Yin}^{2}$ \\ ${ }^{1}$ Pediatric Surgery Department, ${ }^{2}$ Clinical Pharmaceutics Room, Binzhou People's Hospital, Binzhou, 256600, PR China \\ *For correspondence: Email: hahhantjpr@163.com
}

Received: 17 February 2016

Revised accepted: 9 July 2016

\begin{abstract}
Purpose: To explore the clinical effects of low-molecular-weight heparin (LMWH) combined with ulinastatin (UTI) in children with acute pancreatitis.

Methods: In total, 560 patients with severe acute pancreatitis treated at Binzhou People's Hospital, Shandong, China, from April 2012 to June 2014 were enrolled in this study. They were divided into control (280 patients, ulinastatin + conventional treatment) and observational groups (280 patients, $L M W H+$ ulinastatin + conventional treatment). The treatment lasted for 2 weeks. Clinical parameters, laboratory test indices, Acute Physiology and Chronic Health Evaluation (APACHE II) score, and computed tomography score of pancreatic necrosis (CTSPN) were assessed in both groups.

Results: On admission, no significant differences were noted in clinical features, laboratory parameters, APACHE II scores, or CTSPN between the two groups (all $p>0.05$ ). After 2 weeks of treatment, serum amylase, urine amylase, prothrombin time, fibrinogen, partial thromboplastin time, and platelet count in the study group were $913 \pm 281 \mathrm{U} / \mathrm{L}, 1893 \pm 295 \mathrm{U} / \mathrm{L}, 16 \pm 1.60 \mathrm{~s}, 3 \pm 0.60 \mathrm{~g} / \mathrm{L}, 39.80 \pm 5.60 \mathrm{~s}$, and 294 $\pm 49 \times 10^{9} / \mathrm{L}$, respectively, all of which were similar or superior to those in the control group (1738 \pm 346 $U / L, 2453 \pm 473 U / L, 15 \pm 1.50 \mathrm{~S}, 2.50 \pm 0.50,39.80 \pm 5.90$, and $\left.\left.192 \pm 37 \times 10^{9} / \mathrm{L}\right)\right)$. APACHE II scores and CTSPN after 2 weeks of treatment in the observation group were $8.50 \pm 1.80$ and $2.10 \pm 1$, respectively, which were superior to those in the control group $(9.60 \pm 2.40$ and $4.30 \pm 2.60$, respectively; $p<0.05)$. Moreover, the incidence of complications, mortality rate, and average duration of the hospital stay in the observation group were lower than those in the control group $(p>0.05)$. The cure rate in the observation group was higher than that in the control group.

Conclusions: LMWH combined with UTI enhances the efficacy of conventional treatment and reduces mortality. Thus, it is a potentially effective treatment strategy for severe acute pancreatitis in children.
\end{abstract}

Keywords: Acute pancreatitis, Low-molecular-weight heparin, Multiple organ function syndrome, APACHE I/ score, Pancreatic necrosis

\begin{abstract}
Tropical Journal of Pharmaceutical Research is indexed by Science Citation Index (SciSearch), Scopus, International Pharmaceutical Abstract, Chemical Abstracts, Embase, Index Copernicus, EBSCO, African Index Medicus, JournalSeek, Journal Citation Reports/Science Edition, Directory of Open Access Journals (DOAJ), African Journal Online, Bioline International, Open-J-Gate and Pharmacy Abstracts
\end{abstract}

\section{INTRODUCTION}

Severe acute pancreatitis is a special category of acute pancreatitis with multiple complications and a high mortality rate, accounting for 10 to 20 $\%$ of all cases of acute pancreatitis [1]. Severe paediatric acute pancreatitis, which is rarely seen, can be induced by multiple factors and usually presents with symptoms of acute or intermittent abdominal pain. It is easily ignored or misdiagnosed at an early stage, and non-surgical therapy remains the major treatment $[2,3]$. It has been found that effectively inhibiting the activation and release of pancreatin, cell factors, and inflammatory factors; improving the microcirculation; and adjusting the internal environment in the early period are of great 
significance in the prognosis of severe acute pancreatitis [4].

In recent years, many researchers have explored treatment methods for severe acute pancreatitis. In traditional Chinese medicine, researchers have usually chosen to use Rheum officinale, Salvia miltiorrhiza, and peach seed to treat severe acute pancreatitis. In Western medicine, various new drugs have emerged, such as enzyme inhibitors (e.g., octreotide and somatostatin), anti-pancreatin drugs, and cytokine inhibitors (e.g., lexipafant).

However, the curative effects of these drugs are unsatisfactory. Thus, the search for new therapies remains a focus of pancreatitis research. Ulinastatin (UTI) is a broad-spectrum trypsin inhibitor and can slow disease progression by effectively restraining the secretion of pancreatic tissue enzyme and reducing the dissolution and damage to pancreatic tissue by pancreatin [5]. Lowmolecular-weight heparin (LMWH) can improve the microcirculation by inhibiting the activity of thrombin and blood coagulation factors as well as platelet aggregation and reducing the risk of a temporary platelet clot transforming into a more permanent fibrous clot. Moreover, it can reduce the expression of proinflammatory factors and nuclear factor-kB (NF-kB) induced by endotoxins. Its anti-inflammatory effects may include downregulating the expression of inflammatory mediators by lowering the expression of adhesion molecules [6].

Few studies have addressed the application of UTI and LMWH in treating severe paediatric acute pancreatitis. Thus, to further assess the clinical effects of LMWH in combination with UTI in treating severe paediatric acute pancreatitis, 560 children with severe acute pancreatitis were selected for a randomised, controlled study.

\section{METHODS}

\section{Patients}

In total, 560 children with severe acute pancreatitis who were treated in Binzhou People's Hospital from April 2012 to June 2014 were selected for this study. All children satisfied the diagnostic and classificatory criteria for acute pancreatitis [7], and children who developed other chronic diseases, dysfunction in vital organs, and had drug contraindications were excluded. The patients comprised 332 boys and 228 girls aged 2 to 10 years (average, $5.31 \pm$
2.23 years). They were divided randomly into a control group (UTI group) and an observation group (LMWH + UTI group), with 280 children in each group.

Family members of all patients provided written informed consent. This study was approved by the medical ethics committee of Binzhou People's Hospital (approval no. HAH20150623BZ) and conformed to the principles of the Declaration of Helsinki [8].

\section{Evaluation of APACHE II and CTSPN indices}

The Acute Physiology and Chronic Health Evaluation (APACHE II) scoring system consists of an acute physiological score, which includes 12 physiological parameters, an age score, and chronic health conditions. A higher score indicates a more severe disease condition. The computed tomography score of pancreatic necrosis (CTSPN) consists of the computed tomography severity index (CTSI), which equals the grade of acute pancreatitis + the degree of pancreatic necrosis. Severity was divided into three levels: level I ( $0-3$ points), level II (4 - 6 points), and level III (7 - 10 points). Levels II and III are considered severe. Changes in laboratory indexes, including serum amylase (AMS), urine AMS, prothrombin time (PT), fibrinogen (FIB), partial thromboplastin time (PTT), platelet count (PLT), the clinical curative status, and the occurrence of complications were also observed.

\section{Evaluation criteria for curative effect}

The patients were considered cured if their severe acute pancreatitis-related symptoms and abnormal vital signs resolved and associated examination results returned to normal levels. The treatment was deemed effective if the severe acute pancreatitis-related symptoms and vital signs showed differing degrees of improvement and associated examination results basically recovered to normal levels. However, the treatment was deemed ineffective if the severe acute pancreatitis-related symptoms, abnormal vital signs, and associated examination results showed no obvious changes; the disease continued to progress; or the patient died. The following formula was used to calculate the cure rate: (number of cured cases + number of effective cases) / total number of cases.

\section{Statistical analysis}

SPSS software (ver. 19.0, IBM Corp, Armonk, NY, USA) was used to analyse the data. Data are expressed as mean \pm standard deviation. Comparisons between groups were performed 
using the t-test, and comparisons of parameters before and after treatment were performed used the paired t-test. Differences were considered statistically significant at $p<0.05$.

\section{RESULTS}

\section{Comparison between groups}

There were 280 patients in the observation group (178 boys, 102 girls; age, 3 - 9 years; average, $5.14 \pm 2.13$ years) and 280 patients in the control group (154 boys, 126 girls; age, 2 - 10 years; average, $5.25 \pm 2.32$ years). Differences in general data, such as sex and age, between the groups were not statistically significant $(p>$ 0.05). Thus, the groups were comparable.

\section{Changes after treatment}

The APACHE II scores and CTSPN at admission were not significantly different between the groups $(p>0.05)$. After 2 weeks of treatment, the APACHE II scores and CTSPN in the observation group were significantly lower than those in the control group $(p<0.05)$ (Table 1).

\section{Changes in biochemical parameters}

There was no significant difference $(p>0.05)$ in the blood or urine AMS between the two groups on admission. After 2 weeks of treatment, AMS in the observation group was clearly lower than that in the control group $(p<0.01)$. Differences in PT, FIB, PTT, and PLT between the two groups showed no statistical significance $(p>0.05)$ when patients were admitted to the hospital. After 2 weeks of treatment, FIB in the control group decreased markedly. There was no disturbance in blood coagulation in either group (Table 2).

\section{Complications}

Several complications occurred in the control group during treatment: $92(32.90 \%)$ cases of acute respiratory distress syndrome (ARDS); 26 $(9.20 \%)$ cases of mild upper gastrointestinal haemorrhage, of which $12(4.20 \%)$ involved abdominal and subcutaneous haemorrhage; and $56(21.50 \%)$ cases of failure of two or more organs.

Table 1: Changes in APACHE II scores and CTSPN

\begin{tabular}{ccccc}
\hline \multirow{2}{*}{ Group } & \multicolumn{2}{c}{ APACHE II score } & \multicolumn{2}{c}{ CTSPN } \\
\cline { 2 - 5 } & Initial & $\begin{array}{c}\text { After 2-week } \\
\text { treatment }\end{array}$ & Initial & $\begin{array}{c}\text { After 2-week } \\
\text { treatment }\end{array}$ \\
\hline A & $11.60 \pm 3.60$ & $8.50 \pm 1.80$ & $5.40 \pm 1.90$ & $2.10 \pm 1$ \\
$B$ & $11.50 \pm 3.40$ & $9.60 \pm 2.40^{\pi}$ & $5 \pm 1.50$ & $4.30 \pm 2.60$ \\
$t$ value & 0.239 & 4.339 & 0.934 & 4.396 \\
$p$ value & $>0.05$ & $<0.05$ & $>0.05$ & $<0.05$ \\
\hline
\end{tabular}

Observation group: A; Control group: B. Data are presented as mean \pm standard deviation; ${ }^{*} p<0.05$ vs. before treatment

Table 2: Changes in biochemical parameters before and after treatment

\begin{tabular}{lcccccc}
\hline \multirow{2}{*}{ Group } & \multicolumn{2}{c}{ Blood AMS (U/L) } & \multicolumn{2}{c}{ Urine AMS (U/L) } & \multicolumn{2}{c}{ PT (s) } \\
\cline { 2 - 6 } & Initial & $\begin{array}{c}\text { After 2-week } \\
\text { treatment }\end{array}$ & Initial & $\begin{array}{c}\text { After 2-week } \\
\text { treatment }\end{array}$ & $\begin{array}{c}\text { After 2-week } \\
\text { Ireatment }\end{array}$ \\
\hline $\mathrm{A}$ & $3629 \pm 963$ & $913 \pm 281^{*}$ & $5677 \pm 981$ & $1893 \pm 295$ & $15 \pm 1.80$ & $16 \pm 1.60$ \\
$\mathrm{~B}$ & $3684 \pm 895$ & $1738 \pm 346^{*}$ & $5723 \pm 993$ & $2453 \pm 473^{*}$ & $15 \pm 1.50$ & $15 \pm 1.50$ \\
t-value & 0.571 & 11.977 & 0.429 & 11.886 & 0.491 & 5.395 \\
$P$-value & $>0.05$ & $<0.05$ & $>0.05$ & $<0.05$ & $>0.05$ & $<0.05$ \\
\hline
\end{tabular}

\begin{tabular}{lcccccc}
\hline Group & \multicolumn{2}{c}{ FBI (g/L) } & & PTT (s) & \multicolumn{2}{c}{ PLT ( $\left.\times 10^{9} / \mathrm{L}\right)$} \\
\cline { 2 - 7 } & Initial & $\begin{array}{c}\text { After 2-week } \\
\text { treatment }\end{array}$ & Initial & $\begin{array}{c}\text { After 2-week } \\
\text { treatment }\end{array}$ & Initial & $\begin{array}{c}\text { After 2-week } \\
\text { treatment }\end{array}$ \\
\hline $\mathrm{A}$ & $3.60 \pm 0.90$ & $3 \pm 0.60$ & $36.50 \pm 40$ & $39.80 \pm 5.60$ & $180 \pm 19$ & $294 \pm 49$ \\
$\mathrm{~B}$ & $3.50 \pm 0.70$ & $2.50 \pm 0.50$ & $35.70 \pm 3.90$ & $39.80 \pm 5.90$ & $180 \pm 17$ & $192 \pm 37$ \\
t-value & 0.571 & 11.977 & 0.429 & 11.886 & 0.491 & 5.395 \\
P-value & $>0.05$ & $<0.05$ & $>0.05$ & $<0.05$ & $>0.05$ & $<0.05$ \\
\hline
\end{tabular}

Observation group: A; Control group: B. Data are presented as mean \pm standard deviation; ${ }^{*} p<0.05$ vs. before treatment 
Complications in the observation group included $34(12.10 \%)$ cases of ARDS, 10 (3.60 \%) cases of upper gastrointestinal haemorrhage, and 24 $(8.50 \%)$ cases of failure of two or more organs. Thus, the incidence of organ failure and ARDS was significantly higher in the control than observation group $(p<0.05)$.

\section{Comparison of clinical efficacy}

The recovery rate in the control group $(64.30 \%$, $180 / 280$ ) was clearly lower than that in the observation group (86.40\%, 242/280; $p<0.05)$. In the control group, 30 patients underwent surgery (including necrotic pancreatic tissue removal) and 16 patients underwent abdominal washing and drainage. In the observation group, 12 patients underwent surgery and 2 underwent abdominal washing and drainage. The difference in surgery rates between the groups was statistically significant $(p>0.05)$. The mortality rate in the control group $(28.60 \%, 80 / 280)$ was much higher than that in the observation group $(10 \%, 28 / 280)$. Deaths in both groups were the result of multiple organ failure. The average hospital stay in the control group was $43 \pm 11$ days, and that in the observation group was $30 \pm$ 8 days $(p<0.05)$.

\section{DISCUSSION}

Although research on the pathogenesis and mechanisms of severe acute pancreatitis has progressed in recent years, the primary cause of the high mortality remains unknown. Severe acute pancreatitis develops gradually, progressing from autodigestion and free radical injury to excessive activation of white blood cells, secondary strike, and microcirculatory disturbances $[9,10]$.

Currently, several researchers consider inflammatory mediators and microcirculatory disturbances to play important roles in the occurrence and development of severe acute pancreatitis, which provides a theoretical basis for the use of anti-coagulation drugs. UTI, a Kunitz-type broad-spectrum protease inhibitor, has two incompletely overlapping functional areas and can inhibit the activity of multiple proteases and hydrolytic enzymes [11]. UTI can stabilise the activity of enzymes in the cell membrane, regulate ion disorders inside and outside cells, prevent overload of calcium ion inside cells, improve blood perfusion in local tissues, and recover electrical activity and normal form and function in damaged tissues and organs [12]. Moreover, UTI can prevent liver and renal function damage, reduce systemic inflammatory reactions, and increase the success rate of surgery in patients with severe acute pancreatitis in the perioperative period [13].

As an anticoagulation drug, LMWH can effectively restrain the activity of thrombin and blood coagulation factor $\mathrm{Xa}$, inhibit platelet aggregation, and improve the microcirculation. LMWH can also reduce inflammation by lowering the expression of pro-inflammatory factors, inflammatory factors, adhesive factors, and NF$\mathrm{KB}$ induced by endotoxins [14]. Experimental and clinical studies $[15,16]$ have found that LMWH can lower the expression of tumour necrosis factor- $\alpha$ and interleukin-6; reduce the risk of microthrombus formation; relieve microcirculatory issues in the pancreas, kidney, lung, and brain; and lower the fatality rate in patients with severe acute pancreatitis by down-regulating the expression of serum endothelin-I and the activity of NF-KB. Mishreki et al [17] compared the curative effects and safety of LMWH and unfractionated heparin in the treatment of deep venous thrombosis and found that LMWH had a better curative effect and better safety.

In this study, the curative effects of the combination of the two drugs and UTI alone in the treatment of severe acute pancreatitis were compared. The results showed obvious improvements in laboratory indices, much higher cure rate, and lower incidence of complications in the observation than in the control group, suggesting that LMWH plus UTI is safe and effective for treatment of severe acute pancreatitis. In the early stages of severe acute pancreatitis, LMWH could reduce white blood cell-induced peroxidation damage and cell factors by improving the microcirculation and decreasing the adhesion and transfer of white blood cells into tissues. In later stages, the antiinflammatory effects of UTI affected the connection between inflammatory factors and white blood cells, stopped disease progression, improved the microcirculation, and prevented shock, ischaemia-reperfusion injury, and multiple organ dysfunction syndrome.

Furthermore, the results showed that the decline in APACHE-II scores in the observation group was larger than that in the control group. APACHE-II score is an optimal index for evaluating the development of acute pancreatitis and the touchstone of clinical comparative studies [18]. Karimgani et al [19] observed APACHE-II scores in 26 patients with acute pancreatitis on admission and at $48 \mathrm{~h}$ and found a significant difference between the survival and death groups $(6.90 \pm 0.80$ vs. $13 \pm 2.50$ and 8.90 
\pm 1.10 vs. $16.50 \pm 3$, respectively; $p<0.05$ ) Because vital organs are involved in severe pancreatitis, APACHE-II scores show an obvious increase in the early stage $(0-48 \mathrm{~h})$ and the increase becomes faster at 24 to $48 \mathrm{~h}$. A continued increase in APACHE-II scores after discharge usually indicates disease progression. In this study, the APACHE-II scores in the observation group were much lower than those in the control group after treatment, suggesting that the combination of LMWH plus UTI can relieve severe acute pancreatitis-related inflammation and reduce the incidence of complications.

Balthazar et al [20] proposed CTSI criteria in 1990. The CTSI may reflect peripancreatic effusion as well as the degree of pancreatic necrosis. Moreover, it can be used to reflect the incidence of complications and the death rate in patients with acute pancreatitis. Leung et al [21] considered that a CTSI of $\geq 5$ points indicates an increase in the incidence of complications, an extension of the average length of hospital stay, and an increase in the death rate of patients with severe acute pancreatitis. The CTSI is a useful tool for evaluating the severity and prognosis of severe acute pancreatitis, and it is more sensitive than the APACHE-II score. This study found that the CTSI scores of patients in the two groups showed no significant difference on admission, but after 2 weeks of treatment, the CTSI in both groups declined; the decrease in the observation group was more obvious than that in the control group. This suggests that UTI in combination with LMWH was effective in relieving pancreatic and peripancreatic lesions.

\section{Limitations of the study}

This study found that UTI in combination with LMWH had a marked effect in the treatment of acute pancreatitis. However, the difference in surgery rate between the groups was not statistically significant, due possibly to the small sample size. Thus, larger studies are needed to confirm these results.

\section{CONCLUSION}

This study found that UTI, which inhibits pancreatic enzyme secretion, and LMWH, which improves microcirculation, are two important drugs in non-surgical treatment of acute pancreatitis. The two drugs act at different stages, significantly improving the curative effect. UTI in combination with LMWH can rapidly relieve abdominal pain, reduce complications, shorten the length of hospital stay, and enhance the cure rate. All of these effects are of great significance.

\section{DECLARATIONS}

\section{Conflict of Interest}

No conflict of interest associated with this work.

\section{Contribution of Authors}

The authors declare that this work was done by the authors named in this article and all liabilities pertaining to claims relating to the content of this article will be borne by them.

\section{REFERENCES}

1. Li Q, Zhao JJ, Zhu Xi, Yao GQ. Prognostic factors of death in patients with severe acute pancreatitis. CJMIS 2010; 10(9): 816-818.

2. Kang LL. Analysis of clinical effect of treating acute pancreatitis with somatostatin and pantoprazole. Guide China Med 2013; 11(19): 178-179.

3. Zhang $Y, X u X$. Analysis of misdiagnosis of 12 children with acute pancreatitis. China \& Foreign Med Treat 2010; 29(13): 192.

4. Karsidag T, Tüzǜn S, Kemik AS, Purisa S, Unlu A. Alpha1 protease inhibitor and antichymotrypsin levels in acute pancreatitis. Ulus Travma Acil Cerrahi Derg 2012; 18(3): 195.

5. Yilmaz M, Topsakal S, Herek O, et al. Effects of etanercept on sodium taurocholate-induced acute pancreatitis in rats. TransI Res 2009; 154(5): 241.

6. Loira-Pastoriza C, Sapin- Minet A, Diab R, Grossiord JL, Maincent $P$. Low molecular weight heparin gels, based on nanoparticles, for topical delivery. Int J Pharm 2012; 426(1- 2): 256.

7. Pancreatology Group of Surgical Society of Chinese Medical Association. Clinical diagnosis and grading criteria of acute pancreatitis. Chin J Surg 1997; 35(12): 773.

8. Declaration of Helsinki. The 59th World Medical Conference, 2008.

9. Wachira JK, Jensen CH, Rhone K. Doxycycline-induced pancreatitis: a rare finding. SD Med 2013 6(6): 227-229.

10. Zhang YL. Importance of re-understanding the pathogenetic mechanism of acute pancreatitis. Chin $J$ Pract Surg 2003; 23(1): 29-30.

11. Inoue $K$, Takano $H$. Urinary trypsin inhibitor as a therapeutic option for endotoxin- related inflammatory disorders. Expert Opin Investig Drugs 2010; 19(4): 513.

12. Xiao N, Li S, Zhang X, Chen SY. Effect of ephedrine on neuronal plasticity of hypoxic-ischemic brain damage in neonatal rat. Neurosci Lett, 2008; 18(2): 99-102.

13. Zhu FQ, Huang $X L$, SU $Q$, Li R. The application of ulinastatin in severe acute pancreatitis in perioperative period. Harbin Med J 2007; 4: 20-21.

14. Hochart $H$, Jenkins PV, Smith OP, White B. Lowmolecular weight and unfractionated heparins induce a downregulation of inflammation: decreased levels of 
proinflammatory cytokines and nuclear factor-kappaB in LPS-stimulated human monocytes. Br J Haematol 2006; 133(1): 62.

15. Zerem E. Treatment of severe acute pancreatitis and its complications. World J Gastroenterol 2014; 20(38): 13879-13892.

16. Hashimoto $D$, Ohmuraya $M$, Hirota $M$, Yamamoto $A$, Suyama K, Ida S, Okumura Y, Takahashi E, Kido H, Araki K, Baba $H$, Mizushima N, Yamamura $K$. Involvement of autophagy in trypsinogen activation within the pancreatic acinar cells. J Cell Biol 2008 (181): 1065-1072.

17. Mishreki AP, Bowles MJ. A case of widespread aortic thrombosis secondary to acute severe pancreatitis. Ann $R$ Coll Surg Engl 2011; 93(4): e17-18.
18. Bosscha K, Reijinders K, Hulstaert PF, Algra A, Van derWerken C. Prognostic scoring systems to predict outcome in peritonitis and intraabdominal sepsis. $\mathrm{Br} \mathrm{J}$ Surg 1997; 84(11): 1532-1534.

19. Karimgani I, Porter KA, Langevin RE, Banks PA. Prognostic factors in sterile pancreatic-necrosis. Gastroenterology 1992; 103(5):1636-1640

20. Balthazar EJ. Complications of acute pancreatitis: clinical and CT evaluation (Review). Radiol Clin North Am 2002; 40: 1211-1227.

21. Leung TK, Lee CM, Lin SY. Balthazar computed tomography severity index is superior to Ranson criteria and APACHE II scoring system in predicting acute pancreatitis outcome. World J Gastroenterol 2005; 11(38): 6049-6052. 